\title{
BIORDER-PRESERVING COEXTENSIONS OF FUNDAMENTAL SEMIGROUPS
}

\author{
by DAVID EASDOWN
}

(Received 5th February 1987)

\section{Introduction}

In any extension theory for semigroups one must determine the basic building blocks and then discover how they fit together to create more complicated semigroups. For example, in group theory the basic building blocks are simple groups. In semigroup theory however there are several natural choices. One that has received considerable attention, particularly since the seminal work on inverse semigroups by Munn ([14, 15]), is the notion of a fundamental semigroup. A semigroup is called fundamental if it cannot be "shrunk" homomorphically without collapsing some of its idempotents (see below for a precise definition).

For example, Munn showed how all fundamental inverse semigroups can be constructed from semilattices, and proved that any inverse semigroup is a coextension of a fundamental inverse semigroup which possesses the same semilattice of idempotents. (A semigroup $S$ is called a coextension of a semigroup $T$ if $T$ is a homomorphic image of $S$.) This work has been generalized by several authors to wider classes of semigroups $([12,1,11,16,4])$.

The idempotents of an arbitrary semigroup form a biordered set. (Regular biordered sets form the basis for Nambooripad's generalization of Munn's results to regular semigroups). One might ask whether an arbitrary semigroup is a coextension of a fundamental semigroup possessing the same biordered set of idempotents. The following example shows the answer is negative.

Let $S=\left\langle e, f \mid e^{2}=e, f^{2}=f\right\rangle$, the free semigroup on two idempotent generators, so elements of $S$ may be regarded as strings of alternating $e$ 's and $f$ 's. If $\sigma$ is any nontrivial congruence on $S$ then $v \sigma w$ for some $v, w \in S$ where $v \neq w$. Then $(e v f) \sigma(e w f)$ and (fve) $\sigma(f w e)$, so either $(e f)^{i} \sigma(e f)^{j}$ or $(f e)^{i} \sigma(f e)^{j}$ for some natural numbers $i, j$ where $i \neq j$. Hence some power of $e f$ or $f e$ is idempotent in $S / \sigma$. If $S$ and $S / \sigma$ possess the same biordered sets of idempotents, then in $S / \sigma$ we must have some power of ef or $f e$ equalling $e$ or $f$, in which case $e-f, e \rightarrow f, f \sim e$ or $f \rightarrow e$. (" $\sim$ " means "is a left zero of" and " $\rightarrow$ " means "is a right zero of"). Thus the relationships between the idempotents of $S / \sigma$ are different to those of idempotents of $S$, contradicting that $S$ and $S / \sigma$ have the same biordered sets. Hence the biordered set of $S$ cannot be preserved by taking a proper homomorphic image.

However, below we show that every semigroup is a coextension of a fundamental semigroup in which the biordered set of idempotents of the original semigroup has been preserved as a biordered subset. 
For example the three element semilattice<smiles>[CH][Po][Te]C</smiles>

is a fundamental image of the semigroup $S$ defined in the previous paragraph of which the idempotents of $S$ form a biordered subset.

Note by Zorn's Lemma, any semigroup $S$ possesses a maximal idempotent-separating congruence $\sigma$ so $S / \sigma$ is fundamental and the natural map $\sigma \triangleright$ is one-one on idempotents of $S$. However there is no guarantee that $\sigma^{\perp}$ preserves the relationships between the idempotents. For example if $S$ is as defined earlier and $T$ is the two element right-zero semigroup then $T$ is a fundamental image of $S$ with the same number of idempotents, but the biordered set of $S$ has not been preserved, even as a biordered subset.

\section{Preliminaries}

Basic terminology and facts about semigroups and Green's relations, as given in [13] or [2], will be assumed. For terminology and definitions relating to biordered sets of semigroups the reader should consult [5, Section 2]. If $S$ is a semigroup denote its biordered set of idempotents by $E(S)$ and its set of regular elements by $\operatorname{Reg}(S)$. Call a congruence $\sigma$ on $S$ idempotent-separating if

$$
(\forall e, f \in E(S)) e \sigma f \Rightarrow e=f
$$

Call $S$ fundamental if $S$ possesses no idempotent-separating congruences other than the trivial congruence $1_{s}=\{(x, x) \mid x \in S\}$. Call a congruence $\sigma$ on $S$ an $\mathscr{H}$-congruence if

$$
(\forall e \in E(S))(\forall x \in S) e \sigma x \Rightarrow H_{e} \leqq H_{x}
$$

Thus $\mathscr{H}$-congruences are idempotent-separating.

Let $X$ be the set of all regular $\mathscr{L}$-classes of a semigroup $S, Y$ the set of all regular $\mathscr{R}$ classes and $\infty$ a new symbol, and define the representation $\phi=\left(\rho, \lambda^{*}\right)$ by

$$
\begin{aligned}
& \rho: S \rightarrow \mathscr{T}(X \cup\{\infty\}), \text { where, for } s \in S, \\
& \rho_{s}: L_{x} \mapsto \begin{cases}L_{x s} & \text { If } x \mathscr{R} x s \\
\infty & \text { otherwise }\end{cases} \\
& \infty \mapsto \infty
\end{aligned}
$$

and

$$
\lambda: S \rightarrow \mathscr{T}(Y \cup\{\infty\}) \text { where, for } s \in S,
$$




$$
\begin{aligned}
\lambda_{s}: R_{x} \mapsto \begin{cases}R_{s x} & \text { if } x \mathscr{L} s x \\
\infty & \text { otherwise }\end{cases} \\
\infty \mapsto \infty
\end{aligned}
$$

This representation (due to T. E. Hall) first appeared in [5] and [6], though similar representations had been exploited earlier in the literature (see for example [12] or [11]). Set

$$
\begin{aligned}
\mu=\mu(S)= & \operatorname{ker} \phi \\
= & \{(a, b) \in S \times S \mid \text { if } x \in S \text { is regular then each of } \\
& x \mathscr{R} x a, x \mathscr{R} x b \text { implies } x a \mathscr{H} x b \text { and each of } \\
& x \mathscr{L} a x, x \mathscr{L} b x \text { implies } a x \mathscr{H} b x\}
\end{aligned}
$$

This congruence has been studied extensively by Edwards in $[6,7,8,9$ and 10]. He proves

Theorem 1 ([7, Theorem 1] and see also $[3,3.2])$. The congruence $\mu$ is the maximum $\mathscr{H}$-congruence on $S$.

Call a congruence $\sigma$ on a semigroup $S$ biorder-preserving if $E=E(S)$ is a biordered subset of $E(S / \sigma)$ and the natural map $\sigma$. induces an isomorphism from $E$ onto its image $E \sigma$. In this case say $S$ is a biorder-preserving coextension of $S / \sigma$. Equivalently, $\sigma$ is biorder-preserving if and only if

$$
\begin{array}{r}
(\forall e, f \in E) e \rightarrow f \Leftrightarrow e \sigma \rightarrow f \sigma \\
e \curvearrowleft f \Leftrightarrow e \sigma \curvearrowleft f \sigma
\end{array}
$$

Theorem $2[5$, Theorem 6]. The congruence $\mu$ is biorder-preserving.

Theorem $3\left[\right.$, Theorem 8]. For any semigroup $S, \mu(S / \mu(s))=1_{S / \mu(S)}$.

\section{Biorder-preserving coextensions of fundamental semigroups}

The main result is

Theorem 4. Let $S$ be a semigroup for which $\mu=1$. Then $S$ is fundamental.

Proof. Let $\sigma$ be any congruence on $S$ which is idempotent-separating. We show $\sigma$ is an $\mathscr{H}$-congruence, for then $\sigma \subseteq \mu$ by Theorem 1 , so $\sigma=1_{s}$ and we are done.

Suppose then $e \in E(S), x \in S$ and $e \sigma x$. We show $H_{e} \leqq H_{x}$. It is sufficient to show $\phi_{x^{3}}$ is 
idempotent, for then $x^{3} \in E(S)$, since $\phi$ is an isomorphism, so that $e=x^{3}$, since $e \sigma x^{3}$ and $\sigma$ is idempotent-separating.

We show $\rho_{x^{3}}$ is idempotent. If $L \rho_{x^{3}}=\infty$ then $L \rho_{x^{3}}=L \rho_{x^{3}} \rho_{x^{3}}$. Suppose then $L \rho_{x^{3}} \neq \infty$, so $L=L_{y}$ for some $y \in E(S)$, and $y \mathscr{R} y x^{3}$. Hence $y=y x^{3} z$ for some $z \in S$. It is easy to check both $x^{2} z y x$ and $x z y x^{2}$ are idempotent. But $e \sigma x$, so $x \sigma x^{2}$, which gives $\left(x^{2} z y x\right) \sigma\left(x z y x^{2}\right)$. Hence $x^{2} z y x=x z y x^{2}$, since $\sigma$ is idempotent-separating. We have the following egg-box diagram.

\begin{tabular}{|c|c|c|c|}
\hline$y=y x^{3} z$ & $y x$ & $y x^{2}$ & $y x^{3}$ \\
\hline$x^{2} z y$ & $x^{2} z y x$ & $x^{2} z y x^{2}$ & \\
\hline$x z y$ & $x z y x$ & ${ }_{x z y x^{2}}$ & \\
\hline
\end{tabular}

Hence $y x \mathscr{H} y x^{2}$, so by Green's Lemma $y x \mathscr{H} y x^{n}$ for all $n \in \mathbb{N}$. In particular $y x^{6} \mathscr{H} y x^{3}$, so $L \rho_{x^{6}}=L \rho_{x^{3}}$.

This shows $\rho_{x^{3}}$ is idempotent. Dually $\lambda_{x^{3}}$ is idempotent, so also is $\phi_{x^{3}}$, and the proof is complete.

The following is immediate by Theorems 2,3 and 4 :

Corollary 5. If $S$ is any semigroup then $S / \mu$ is fundamental and $E(S)$ is a biordered subset of $E(S / \mu)$. Thus every semigroup is a biorder-preserving coextension of a fundamental semigroup.

\section{REFERENCES}

1. A. H. Cuifford, The fundamental representation of a regular semigroup, Semigroup Forum 10 (1975), 84-92.

2. A. H. Clifford and G. B. Preston, The Algebraic Theory of Semigroups, Vol. I (Amer. Math. Soc. Math. Survey 7, Providence, R.I., 1961).

3. D. EAsDown, Biordered Sets of Semigroups (Ph.D. thesis, Monash University, 1983).

4. D. EAsDown, Fundamental Semigroups and biordered sets, (to be sumitted).

5. D. EASDOWN and T. E. HALL, Reconstructing some idempotent-generated semigroups from their biordered sets, Semigroup Forum 29 (1984), 207-216.

6. P. M. Edwards, Eventually regular, semigroups, Bull. Austral. Math. Soc. 28 (1983), 23-38.

7. P. M. Edwards, Fundamental semigroups, Proc. Roy. Soc. Edinburgh 99A (1985), 313-317. 
8. P. M. Edwards, On the lattice of congruences on an eventually regular semigroup, $J$. Austral. Math. Soc. 38 (1985), 281-286.

9. P. M. Edwards, Eventually regular semigroups that are group-bound, Bull. Austral. Math. Soc. 34 (1986), 127-132.

10. P. M. Edwards, Maximal Semigroup Congruences (to be submitted).

11. P. A. GriLlet, The structure of regular semigroups, I: A representation, Semigroup Forum 8 (1974), 177-183.

12. T. E. Hall, On regular semigroups, J. Algebra 24 (1973), 1-24.

13. J. M. Howle, An Introduction to Semigroup Theory (Academic Press, London, 1976).

14. W. D. MunN, Uniform semilattices and bisimple inverse semigroups, Quart. J. Math. Oxford Ser. (2) 17 (1966), 151-159.

15. W. D. Munn, Fundamental inverse semigroups, Quart. J. Math. Oxford Ser. (2) 21 (1970), 152-170.

16. K. S. S. Nambooripad, Structure of regular semigroups, I, Mem. Amer. Math. Soc. 224 (1979).

Department of Mathematics

UNIVERSITY OF WESTERN AUSTRALIA

NeDLANDS WA 6009

Australia 